\title{
Article \\ Equivalent Analysis of Thermo-Dynamic Blow-Off Impulse under X-ray Irradiation
}

\author{
Dengwang Wang ${ }^{1,2}$, Yong Gao ${ }^{1}{ }^{\mathbb{D}}$, Wei Chen ${ }^{2}$, Jing Zhang ${ }^{1}$ and Sheng Wang ${ }^{1, *}$ \\ 1 Department of Nuclear Science and Technology, Xi'an Jiaotong University, Xi'an 710000, China; \\ wdw21s@stu.xjtu.edu.cn (D.W.); gaoyong1108@stu.xjtu.edu.cn (Y.G.); zhangjing1108@stu.xjtu.edu.cn (J.Z.) \\ 2 Northwest Institute of Nuclear Technology, Xi'an 710024, China; chenwei@nint.ac.cn \\ * Correspondence: shengwang@xjtu.edu.cn
}

Citation: Wang, D.; Gao, Y.; Chen, W.; Zhang, J.; Wang, S. Equivalent Analysis of Thermo-Dynamic Blow-Off Impulse under X-ray Irradiation. Appl. Sci. 2021, 11, 8853. https://doi.org/10.3390/app11198853

Academic Editor: Andrea Frazzica

Received: 6 June 2021

Accepted: 20 September 2021

Published: 23 September 2021

Publisher's Note: MDPI stays neutral with regard to jurisdictional claims in published maps and institutional affiliations.

Copyright: (c) 2021 by the authors. Licensee MDPI, Basel, Switzerland. This article is an open access article distributed under the terms and conditions of the Creative Commons Attribution (CC BY) license (https:// creativecommons.org/licenses/by/ $4.0 /)$.

\begin{abstract}
X-ray thermodynamic effect is an important damage mode for spacecraft. Blow-off impulse as the main thermodynamic damage parameter has been widely studied by combining laboratory and numerical simulations. In this paper, most calculations and analyses have been carried out by using the self-developed software RAMA, including the equivalent calculation of blow-off impulse of monoenergetic and blackbody $\mathrm{X}$-ray, and soft/hard blackbody $\mathrm{X}$-ray irradiated at different incidence angles of LY-12 aluminium target. The results show that the characteristic mono-energetic X-ray can be exploited to simulate the blow-off impulse of the blackbody X-ray under certain conditions as a feasible equivalent method for the equal-flux and equal-impulse relations between mono-energetic and intense pulse blackbody of blow-off impulse. Moreover, the equivalent thermodynamic effect can be achieved between the point source radiation and parallel X-ray of X-ray. Furthermore, the cosine distribution of blow-off impulse is conducive to designing and calculating $\mathrm{X}$-ray radiation load of hard aluminium corresponding to $1-5 \mathrm{keV}$ blackbody spectrum. The mentioned results can be referenced for pulse $X$-ray simulation source and enhance the fidelity of the thermal-mechanical effect by electron beam. It is noteworthy that the study on the thermodynamic effects of intense pulsed X-ray is of high significance.
\end{abstract}

Keywords: blow-off impulse; equivalence; angle of incidence; thermo-dynamics; energy deposition

\section{Introduction}

Since the 1950s and 1960s, the study on multiple effects of X-ray has aroused extensive attention, especially on X-ray thermodynamic effects as a basic mean of spacecraft damage [1-3] has aroused extensive attention. In terms of the mentioned effects, a combination of laboratory and numerical simulations is required [4-6]. In the past half century, people have extensively developed a variety of simulation experimental equipment, such as lowenergy relativistic electron beam accelerator $[7,8]$, etc., as well as a series of related software in terms of calculation [9-11]. These studies play a very important role in the development and application of anti-X-ray reinforcement technology $[12,13]$.

The intense pulsed $X$-ray can exert a robust damaging effect [14]. When a certain intensity of pulsed X-ray irradiates the medium surface, X-ray energy will suddenly deposit on the surface of this material with a certain thickness, and the material temperature will rise and expand rapidly to excite robust stress wave, which is termed as thermal shock wave [15]. In the meantime, when the temperature or internal energy reaches a certain value, the material will melt or vaporize [16], which will have effects on the dielectric material at a certain expansion speed and spray outward, with the material or structures exhibiting the form of recoil, which is termed as blow-off impulse. The impulse can induce structural responses (e.g., vibration [17], deformation [18] and buckling of structural shells [19]). Thermal shock wave, blow-off impulse and structural response refer to three basic forms of damaging effect of intense pulse $X$-ray, and these studies on such forms are of high practical significance [20,21]. 
H.L. Yang [22] studied the energy deposition profiles of three mono-energetic electron beams of aluminium at different angles of incidence and found that low-energy electron beams at a large angle could simulate the radiating effect of intense pulse X-ray. Y. Hu [23] reported that electron beam had an angle of incidence distribution in the spot range of the anode target in the study on thermal-mechanical effect of multi-spectral electron beams, and that the distribution of the angle of incidence significantly impacted the energy deposition profile of the multi-spectral electron beam in the target material.

In this study, the X-ray blow-off impulse of two mono-energetic and blackbody spectra on aluminium alloy materials was calculated with self-developed numerical software. The mono-energetic and blackbody $X$-rays in the laboratory were discussed as the equivalent method, and point source was found feasible in simulating parallel light radiation source based on the mentioned results.

\section{Finite Element Calculation Software}

Due to the failure of the existing finite element analysis software to deal with the thermodynamic problems caused by electron beam and pulsed X-ray irradiation, we used the finite element method and the FORTRAN language to compile the program RAMA [24,25] for the thermodynamic effects of pulsed beam irradiation to carry out relevant numerical simulation work. By embedding a variety of constitutive models and equations of state including orthotropic dynamic elastoplastic constitutive model, the RAMA program can not only deal with the fracture and vaporization of materials but simulate the stress wave propagation in the collision of two-dimensional flat plates by anisotropic and isotropic materials, and the thermodynamic effects of two-dimensional X-ray in various shapes [26,27]. It thus has a certain practicality in engineering applications [27].

\subsection{Calculation Process}

The calculation process of electron beam energy deposition can be summarized as follows:

In a unit mass thickness material $S(E)$, the electron energy loss, mainly caused by electron collision ionization for lower-energy electrons, is as follows [28,29]:

$$
S(E)=\frac{2 \pi e^{4} N_{0} Z}{m_{0} c^{2} \beta^{2} A}\left\{\operatorname{In}\left[\frac{m_{0} c^{2} \beta^{2} E}{2 J^{2}\left(1-\beta^{2}\right)}\right]-\left[2\left(1-\beta^{2}\right)^{1 / 2}-1+\beta^{2}\right] \operatorname{In} 2+\left(1-\beta^{2}\right)+\frac{1}{8}\left[1-\left(1-\beta^{2}\right)^{1 / 2}\right]^{2}-\delta_{0}\right\}
$$

where $N_{0}$ is the Avogadro constant, $e$ is the electron charge, $Z$ is the atomic number, $A$ is the atomic weight, $m_{0} c^{2}=0.511 \mathrm{MeV}$ is the electron rest mass, $\beta=\left\{1-\left[m_{0} c^{2} /\left(E+m_{0} c^{2}\right)\right]^{2}\right\}$, $E$ is the kinetic energy of electron, $J(\mathrm{MeV})$ is the average ionization energy, $\delta_{0}$ is the density effect correction factor and $\delta_{0}$ is introduced to reduce energy loss by electron collisions through target polarization.

The next step in electronic energy is decided by the law of energy logarithmic delay

$$
E_{n+1}=K E_{n}
$$

where $K$ is 0.9576 .

The continuous slow-down used to approximately calculate the interval mass range of electron.

$$
\Delta S_{n+1}=\int_{E_{n+1}}^{E_{n}}\left|\frac{d E}{d S}\right|^{-1} d E
$$

The electronic coordinates of step $n+1$ are as follows.

$$
X_{n+1}=X_{n}+\Delta S_{n+1} \frac{\cos \theta_{n+1}}{\rho(j)}
$$

where $\rho(j)$ is the Lagrange interval density of $j . \theta_{n+1}$ is the positive angle between the electron motion direction and the $X$-axis of step $n+1$. 
According to the Moliere Multiple Scattering Theory, the scattering angle is as follows [30],

$$
F(\theta)=\mathrm{f}^{(0)}(\theta)+\mathrm{f}^{(1)}(\theta) / B+\mathrm{f}^{(2)}(\theta) / B^{2}, \omega=X_{c} B^{1 / 2} \theta
$$

where $B$ and $X_{c}$ are the parameters that depends on energy and path length, and it's directly related to energy. $\theta$ is the discrete angle.

The electronic polar angle is as follows.

$$
\theta_{n+1}=\cos ^{-1}\left(\cos \theta_{n} \cos \omega+\sin \theta_{n} \sin \omega \cos \varphi\right)
$$

where $\omega$ is the electronic azimuth, $\varphi$ is the determined uniform sampling.

If the deposition energy of each incident electron is $\Delta E_{i}$ in the interval $J$, the total deposition energy of each incident electron is as follows [8],

$$
E_{n}=\sum_{i=1}^{N} \Delta E_{i}
$$

The specific energy of unit energy flux in interval $J$ is as follow.

$$
Q_{J}=\frac{E_{n}}{\rho_{0} \Delta x N}
$$

where $\Delta x$ is the mesh step.

According to the current and voltage waveform of experiment, the energy deposition of electron beam can be calculated, as shown as below:

$$
E_{R}(\mathrm{x}, \mathrm{t})=U_{t} I_{t} Q_{J} \Delta T / S
$$

where $U_{t}$ and $I_{t}$ are the instantaneous voltage and current of REB diode, $\Delta T$ is the time step, $S$ is the spot area of electron beam on the target.

\subsection{Basic Equation}

As shown in Figure 1, if the X-ray is perpendicular to an axial irradiated cylinder, and the states of each section are assumed to be the same, any physical quantity is independent of the axial coordinate $Z$, then the problem is simplified to be a two-dimensional plane one $[27,28]$.

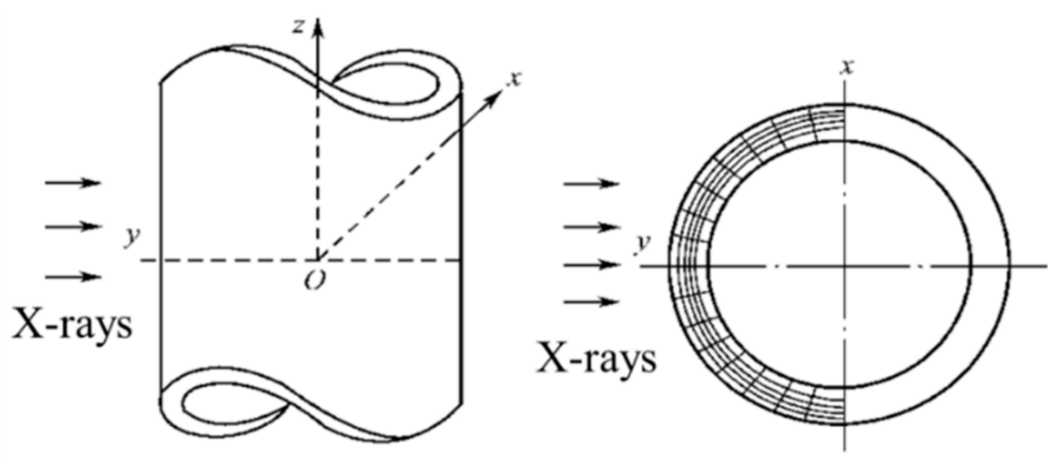

Figure 1. Two-dimensional plane problem.

For a two-dimensional problem,

$$
\varepsilon_{y z}=0, \varepsilon_{x z}=0, \varepsilon_{z z}=0, \sigma_{x z}=0, \sigma_{y z}=0
$$

The symmetric stress tensor and the deformation velocity tensor are as follows [30], 


$$
\boldsymbol{\sigma}=\left(\begin{array}{ccc}
\sigma_{x x} & \sigma_{x y} & 0 \\
\sigma_{x y} & \sigma_{y y} & 0 \\
0 & 0 & \sigma_{z z}
\end{array}\right) \text { and } \dot{\varepsilon}^{\prime}=\left(\begin{array}{ccc}
\dot{\varepsilon}_{x x}^{\prime} & \dot{\varepsilon}_{x y}^{\prime} & 0 \\
\dot{\varepsilon}_{x y}^{\prime} & \dot{\varepsilon}_{y y}^{\prime} & 0 \\
0 & 0 & 0
\end{array}\right)=\left(\begin{array}{ccc}
\frac{\partial u}{\partial x} & \frac{1}{2}\left(\frac{\partial u}{\partial y}+\frac{\partial v}{\partial x}\right) & 0 \\
\frac{1}{2}\left(\frac{\partial u}{\partial y}+\frac{\partial v}{\partial x}\right) & \frac{\partial v}{\partial y} & 0 \\
0 & 0 & 0
\end{array}\right)
$$

The two-dimensional strain irradiation problem satisfies the following conserved elastoplastic hydrodynamic equations in the Euler coordinate system, in the case of ignoring the force and heat conduction [31],

$$
\begin{gathered}
\frac{\partial \boldsymbol{U}}{\partial t}+\frac{\partial \boldsymbol{F}(\boldsymbol{U})}{\partial x}+\frac{\partial \boldsymbol{G}(\boldsymbol{U})}{\partial y}=\boldsymbol{W} \\
\boldsymbol{U}=\left(\begin{array}{c}
\rho \\
\rho u \\
\rho v \\
\rho e_{k}
\end{array}\right), F=\left(\begin{array}{c}
\rho u \\
\rho u^{2}-\sigma_{x x} \\
\rho u v-\sigma_{x y} \\
u\left(\rho e_{k}-\sigma_{x x}\right)-v \sigma_{x y}
\end{array}\right), \boldsymbol{G}=\left(\begin{array}{c}
\rho v \\
\rho u v-\sigma_{x y} \\
\rho v^{2}-\sigma_{y y} \\
v\left(\rho e_{k}-\sigma_{y y}\right)-u \sigma_{x y}
\end{array}\right), \boldsymbol{W}=\left(\begin{array}{c}
0 \\
0 \\
0 \\
\rho Q
\end{array}\right) \\
e_{k}=\left[E+\left(u^{2}+v^{2}\right) / 2\right], \text { and }\left\{\begin{array}{c}
\sigma_{x x}=S_{x x}-p+q \\
\sigma_{y y}=S_{y y}-p+q \\
\sigma_{z z}=S_{z z}-p \\
\sigma_{x y}=S_{x y}
\end{array}\right.
\end{gathered}
$$

where $q$ is the artificial viscosity.

The Lagrange method is used in numerical simulation, and the motion equations is,

$$
\left\{\begin{array}{l}
\rho \frac{\partial u}{\partial t}=\frac{\partial \sigma_{x x}}{\partial x}+\frac{\partial \sigma_{x y}}{\partial y} \\
\rho \frac{\partial v}{\partial t}=\frac{\partial \sigma_{x y}}{\partial x}+\frac{\partial \sigma_{y y}}{\partial y}
\end{array}\right.
$$

where $x$ and $y$ are the Euler space coordinates of the trajectory of Lagrange particles.

When used, the material element will rotate in the plane dynamic stress state and Lagrange coordinates. However, the above equations of motion are given in a fixed coordinate system, so the influence of such rotation should be considered in the numerical calculation. Since the stress-strain relation must be independent of the rigid body motion, the coordinate correction of the stress-strain relation must be carried out in the coordinate system to give the stress at the new position in the coordinate system.

The stress caused by rotation is modified to,

$$
\left\{\begin{array}{c}
\Delta S_{x x}=S_{x x} \cos ^{2} \Delta \alpha+S_{y y} \sin ^{2} \Delta \alpha+2 S_{x y} \sin \Delta \alpha \cos \Delta \alpha-S_{x x} \\
\Delta S_{y y}=S_{x x} \sin ^{2} \Delta \alpha+S_{y y} \cos ^{2} \Delta \alpha-2 S_{x y} \sin \Delta \alpha \cos \Delta \alpha-S_{y y} \\
\Delta S_{x y}=S_{x y}\left(\cos ^{2} \Delta \alpha-\sin ^{2} \Delta \alpha\right)+\left(S_{y y}-S_{x x}\right) \sin \Delta \alpha \cos \Delta \alpha-S_{x y}
\end{array}\right.
$$

Or,

$$
\left\{\begin{array}{l}
\Delta S_{x x}=\frac{S_{x x}-S_{y y}}{S_{x x}-S_{y y}}(\cos 2 \Delta \alpha-1)+S_{x y} \sin 2 \Delta \alpha \\
\Delta S_{y y}=\frac{\cos 2 \Delta \alpha)-S_{x y} \sin 2 \Delta \alpha}{2}(1-\cos 2 \Delta \alpha-1)+\frac{S_{y y}-S_{x x}}{2} \sin 2 \Delta \alpha \\
\Delta S_{x y}=S_{x y}(\cos 2 \Delta x
\end{array}\right.
$$

The rotation stress per unit time is modified as,

$$
\dot{\delta}_{x x}=\frac{\Delta S_{x x}}{\Delta t}, \dot{\delta}_{y y}=\frac{\Delta S_{y y}}{\Delta t}, \dot{\delta}_{x y}=\frac{\Delta S_{x y}}{\Delta t}
$$


And the stress deviation can be written as,

$$
\left\{\begin{array}{c}
\dot{S}_{x x}=2 G\left(\dot{\varepsilon}_{x x}-\frac{1}{3} \frac{\dot{V}}{V}\right)+\dot{\delta}_{x x} \\
\dot{S}_{y y}=2 G\left(\dot{\varepsilon}_{y y}-\frac{1}{3} \frac{\dot{V}}{V}\right)+\dot{\delta}_{y y} \\
\dot{S}_{z z}=2 G\left(-\frac{1}{3} \frac{V}{V}\right) \\
\dot{S}_{x y}=G \dot{\varepsilon}_{x y}+\dot{\delta}_{x y}
\end{array}\right.
$$

The above equation is only valid within the elastic limit.

The yield criterion for two-dimensional plane strain problem is,

$$
\left(\sigma_{x x}-\sigma_{y y}\right)^{2}+\left(\sigma_{x x}-\sigma_{z z}\right)^{2}+\left(\sigma_{y y}-\sigma_{z z}\right)^{2}+6 \sigma_{x y}^{2}=2 \Upsilon_{0}^{2}
$$

This is the same as the following equation,

$$
\left(S_{x x}-S_{y y}\right)^{2}+\left(S_{x x}-S_{z z}\right)^{2}+\left(S_{y y}-S_{z z}\right)^{2}+6 S_{x y}^{2}=2 Y_{0}^{2}
$$

The yield criterion can be simplified as,

$$
S_{x x}^{2}+S_{y y}^{2}+S_{z z}^{2}+2 S_{x y}^{2}=2 J_{0}=\frac{2}{3} Y_{0}^{2}
$$

where $J_{0}>Y_{0}^{2} / 3$, the following substitution shall be made,

$$
\left\{\begin{aligned}
S_{x x} & \rightarrow \sqrt{\frac{1}{3 J_{0}}} Y_{0} S_{x x}, S_{y y} \rightarrow \sqrt{\frac{1}{3 J_{0}}} Y_{0} S_{y y} \\
S_{z z} & \rightarrow \sqrt{\frac{1}{3 J_{0}}} Y_{0} S_{z z}, S_{x y} \rightarrow \sqrt{\frac{1}{3 J_{0}}} Y_{0} S_{x y}
\end{aligned}\right.
$$

The continuity equation of two-dimensional plane problem of Euler form in Cartesian coordinate system is as follows,

$$
\frac{1}{V} \frac{\mathrm{d} V}{\mathrm{~d} t}=-\frac{\partial u}{\partial x}+\frac{\partial v}{\partial y} \text { or } \frac{1}{V} \frac{\mathrm{d} V}{\mathrm{~d} t}=-\frac{\partial u}{\partial x}+\frac{\partial v}{\partial y}
$$

The description in the Lagrange is as follows,

$$
\frac{1}{V} \frac{\partial V}{\partial t}=\frac{\partial u}{\partial x}+\frac{\partial v}{\partial y}
$$

The energy conservation equation of Euler form in the Cartesian coordinate system, is,

$$
\frac{\mathrm{d} E}{\mathrm{~d} t}+(p+q) \frac{\partial V}{\partial t}-V\left[S_{x x} \frac{\partial u}{\partial x}+S_{y y} \frac{\partial v}{\partial y}+S_{x y}\left(\frac{\partial v}{\partial x}+\frac{\partial u}{\partial y}\right)\right]=Q
$$

The description in the Lagrange is as follows,

$$
\frac{\partial E}{\partial t}+(p+q) \frac{\partial V}{\partial t}-V\left[S_{x x} \frac{\partial u}{\partial x}+S_{y y} \frac{\partial v}{\partial y}+S_{x y}\left(\frac{\partial v}{\partial x}+\frac{\partial u}{\partial y}\right)\right]=Q
$$

\subsection{Boundary Conditions and Initial Conditions}

The definite solution of the elastoplastic hydrodynamics equations can be carried out under the boundary conditions which can be divided into free surface boundary conditions and solid wall conditions. The latter is aimed to simplify the calculation because of the symmetry of the object [32].

The free surface boundary condition is as follows,

$$
p=0, S_{x x}=0, S_{y y}=0, S_{z z}=0, S_{x y}=0, q=0
$$


At the free surface boundary, the numerical calculation has unified calculation procedure and format, and the density, stress tensor and other physical quantities are usually used to create a grid of zero.

It is according to the requirement of symmetry that the fixed wall boundary condition requires $u=0$. In addition, in order to have a unified calculation program and format in the numerical simulation, a mirror-reflection grid is constructed at the solid wall boundary, which is mirror-image symmetric with the real grid in the solid wall. In this case, the normal stress component is symmetrically distributed on both sides of the solid wall, the shear stress component is antisymmetric and the density is the same.

The initial condition is as follows,

$t=0, p=0, S_{x x}=0, S_{y y}=0, S_{z z}=0, S_{x y}=0, q=0, u=0, v=0, E=0, V=V_{0}$

\subsection{Artificial Viscosity}

Artificial viscosity for two dimensional problems includes scalar viscosity, tensor viscosity and rotational viscosity [33]. In the numerical simulation of two-dimensional elastoplastic hydrodynamics problems, it can not only solve the continuity calculation of the discontinuity plane and eliminate the post-wave oscillation, but also play an important role in maintaining the deformation stability of the mesh. Therefore, it is more complicated than one-dimensional problems.

The use of scalar viscosity is similar to the one-dimensional problem, that is, a combination of primary viscosity and secondary viscosity is added to the pressure term. Therefore, the scalar artificial viscosity used in two-dimensional numerical simulation is essentially an extension of the one-dimensional artificial viscosity $q_{1}$.

For scalar viscosity in two-dimensional plane problems, the following form is more suitable.

$$
q=\left\{\begin{array}{cl}
\frac{a^{2} A}{V}\left(\frac{1}{V} \frac{\mathrm{d} V}{\mathrm{~d} t}\right)^{2}+\frac{b \sqrt{(\partial p / \partial \rho) A}}{V}\left|\frac{1}{V} \frac{\mathrm{d} V}{\mathrm{~d} t}\right|, & \frac{\partial V}{\partial t}<0 \\
0, & \frac{\partial V}{\partial t} \geq 0
\end{array}\right.
$$

where the value range of adjustable parameters $a$ and $b$ is similar to the one-dimensional irradiation problem, where $A$ is the area of the characteristic grid.

The artificial viscosity of Navier-Stokes can be introduced to eliminate the mesh distortion that may occur in the numerical calculation process, and the deviating stress term can be added in the two-dimensional and three-dimensional elastoplastic hydrodynamics problems. For two dimensional problems, the form is,

$$
\left\{\begin{array}{c}
q_{x x}=2 G_{1}\left(\dot{\varepsilon}_{x x}-\frac{1}{3} \frac{\dot{V}}{V}\right) \\
q_{y y}=2 G_{1}\left(\dot{\varepsilon}_{y y}-\frac{1}{3} \frac{\dot{V}}{V}\right) \\
q_{x y}=G_{1} \dot{\varepsilon}_{x y} \\
G_{1}=\frac{C_{3} \sqrt{A}}{V}
\end{array}\right.
$$

where $c_{3}$ is also the adjustment parameter and $c$ is the local sound velocity for twodimensional problems, and $A$ is the mesh area. Using the above viscosity, Equation (31) should be rewritten as,

$$
\left\{\begin{array}{c}
\dot{S}_{x x}=2\left(G+\frac{G_{1}}{\Delta t}\right)\left(\dot{\varepsilon}_{x x}-\frac{1}{3} \frac{\dot{V}}{V}\right)+\dot{\delta}_{x x} \\
\dot{S}_{y y}=2\left(G+\frac{G_{1}}{\Delta t}\right)\left(\dot{\varepsilon}_{y y}-\frac{1}{3} \frac{\dot{V}}{V}\right)+\dot{\delta}_{y y} \\
\dot{S}_{x y}=\left(G+\frac{G_{1}}{\Delta t}\right) \dot{\varepsilon}_{x y}+\dot{\delta}_{x y}
\end{array}\right.
$$




\subsection{Stability Conditions}

The stability condition of the difference equation for two dimensional problems has many forms, which are usually taken as follows,

$$
\Delta t \leq\left[\frac{\alpha \Delta L}{C}\right]_{j, k} \text { or } \Delta t \leq \alpha \cdot \min \left[\frac{\sqrt{\Delta A}}{C}\right]_{j, k}
$$

where $C$ is the local sound velocity, $\Delta L$ is the minimum characteristic length of the calculated region, $\Delta A$ is the area of the grid cell and $\alpha$ is the corresponding adjustment coefficient, which is $\alpha \approx 0.5 \sim 0.8$.

\subsection{The Energy Limit Point Method of Two-Dimensional Numerical Simulation}

The grid should not be too large according to the accuracy requirements, otherwise, the exponential decay of energy deposition will lead to large errors in the numerical simulation of the two-dimensional problem of pulse beam irradiation. At the same time, the number of photon clusters is usually large in the calculation of energy deposition, so the calculation is time-consuming in energy deposition for two-dimensional problems. In order to save the computerization, the following "energy limit point method" can be used.

The "energy limit point method" [34] is basically to simplify the calculation of energy deposition in some areas according to the characteristics of energy deposition. In fact, since most of the energy of X-rays (especially soft X-rays) is deposited in an extremely small area on the surface, and if such deposition is small in a relatively large depth area of the irradiated material, the thermal stress value generated will be small as well, generally below $1 \mathrm{MPa}$. In these regions, the stress of this magnitude can be ignored without affecting the calculation and analysis of the overall thermal-mechanical effect. Therefore, the corresponding energy deposition in these regions can be ignored.

Energy deposition value $E_{\delta}$ that is allowed to be ignored or not to be calculated can be determined in advance, and its corresponding thermal stress is very small, e.g., about $0.6 \mathrm{MPa}$ for $E_{\delta}=0.1 \mathrm{~J} / \mathrm{g}$ with aluminium. We can always find such a point, $\theta_{j_{0}, k_{0}}$ is the azimuth angle, $r_{j_{0}, k_{0}}$ is the distance from the irradiated surface of the object.

$$
E_{j_{0}, k_{0}}=\int_{0}^{t_{0}} Q\left(r_{j_{0}, k_{0}}\right) \mathrm{d} t \cos \theta_{j_{0}, k_{0}} \approx E_{\delta}
$$

Therefore, the energy deposition of all grid points can't be calculated. The practical calculation shows that the "energy limit point method", which is highly effective, can not only facilitate the calculation of the whole thermal-mechanical effect and the characteristic analysis of radiation, but significantly save machine time.

\section{Analysis of Mesh Independence}

Determined by grid size [9,31,32], the numerical results cannot be changed easily by independent grid, except the encrypted grid that can slightly affect the numerical results. Numerical calculation should be grid-independent, which is also essentially required for the international academic community to accept numerical calculation. Grid-independent results have associations with specific physical problems, which should be determined by trial calculation according to specific problems. Overall, the grid can be calculated from large to small, and it is capable of comprehensively considering the precision and speed of the calculated results. Thus, the appropriate mesh size is critical for numerical calculation.

X-ray energy decays exponentially in the material when irradiating the target. It is primarily deposited as a thin layer on the surface of a material. Furthermore, the X-ray energy deposition profile varies rapidly with the depth of the thin layer. Accordingly, different mesh sizes significantly impact the distribution of the X-ray energy deposition, which critically determines blow-off impulse calculation. The numerical results of blow-off 
impulse are largely determined by grid size, so the irrelevant grid should be developed, and the grid-independent solution should be obtained.

Figure 2 illustrates the result of blow-off impulse when mono-energetic and blackbody $\mathrm{X}$-rays irradiate aluminium target by different mesh sizes. The effect of mesh size on impulse results is obviously shown. Blow-off impulse varies with the melting or gasification of a material, which was not discussed in this study. The mesh size was $0.0005 \mathrm{~cm}$ to impulse and computational efficiency, and the appropriate time step was adopted given the finite difference stability condition.

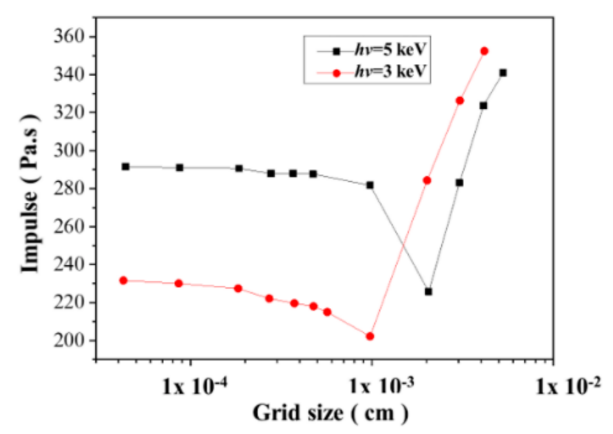

(a)

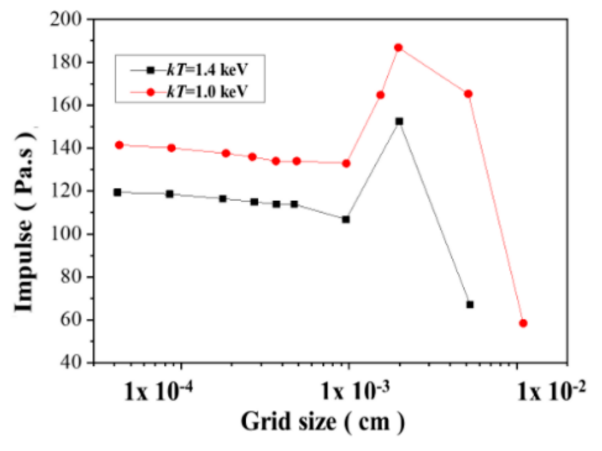

(b)

Figure 2. Effect of grid size on blow-off impulse. (a) Mono-energetic. (b) Blackbody X-rays.

\section{Blackbody Spectrum and Mono-Energy X-ray}

Blackbody spectrum X-ray refers to the energy radiated outward in the form of electromagnetic waves by high temperature objects. The $\mathrm{X}$-ray energy and the proportion of photon component vary with the object temperature. The radiation intensity is computed by the Plank formula.

$$
B_{v}=\frac{2 \pi h v^{3}}{c^{2}} \frac{1}{e^{\frac{h v}{k T}}-1}
$$

where $h$ denotes the Plank constant $\left(h=6.62 \times 10^{-34} \mathrm{~J} \cdot \mathrm{s}\right), v$ is the photon frequency, $c$ is the light speed, $k$ is the Boltzmann constant $\left(k=1.38 \times 10^{-13} \mathrm{~J} / \mathrm{K}\right)$, T represents the blackbody temperature, $h v$ is the photon energy $(\mathrm{keV})$ and $k T$ is the blackbody temperature $(\mathrm{keV})$. Figure 3 shows black-body spectrum radiation intensity at different temperatures.

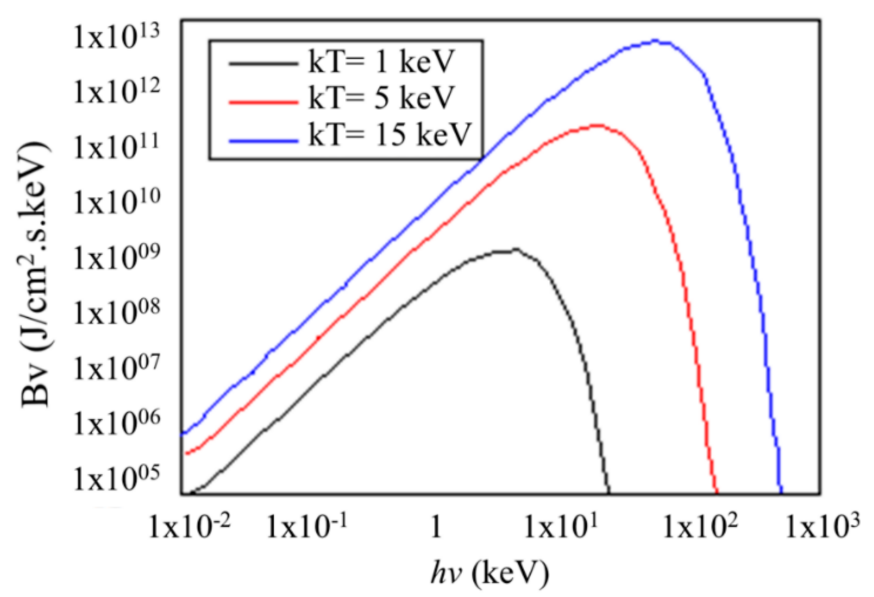

Figure 3. Black-body spectrum radiation intensity at different temperatures.

The normalized spectrum is usually employed practically, and the formula is written as:

$$
b_{v}=\frac{B_{v}}{\sigma T^{4}}=\frac{15 h^{4} v^{3}}{\pi^{4} k^{4} T^{4}} \frac{1}{e^{\frac{h \nu}{k T}}-1}
$$


The proportion of X-ray photons with the energy in the interval between $h v$ and $h v+\Delta h$ is expressed as:

$$
f_{v}=\frac{15(h v)^{3}}{\pi^{4}(k T)^{4}} \frac{\Delta h v}{e^{\frac{h v}{k T}}-1}
$$

Plank distribution reaches its peak (photon energy $h v=2.82 \mathrm{kT}$ ). The proportion of $\mathrm{X}$-ray photos energy $(h v \leq 1 \mathrm{kT})$ is less than $3.5 \%$ of the total radiation energy, and that $(h v \leq 2.82 \mathrm{kT})$ takes up $35 \%$ of the total radiation energy, and that ( $h v \geq 10 \mathrm{kT}$ ) is less than $3.5 \%$ of the total radiation energy. Thus, the mean value energy of the blackbody spectrum is defined as $h v=3.5 \mathrm{kT}$.

X-rays collide with nucleus and extranuclear electrons in a material; as a result, the $X$-ray energy will be deposited inside the material. Macroscopically, the energy deposition of X-ray abides by the Lambert's law, which is defined as:

$$
\Phi=\Phi_{0} e^{-\mu \rho r}
$$

where $\Phi$ denotes the energy flux after X-ray passing through materials (thickness $=r$ ), $\Phi_{0}$ is the incident energy fluxes, $\rho$ is the density, $\mu$ represents the absorption coefficients, which is related to materials and photon energy of X-ray.

It is therefore concluded that the deposited energy of X-ray in the unit mass material at $\delta$ is defined as:

$$
E=\frac{\Phi_{0}}{\rho \delta}\left(1-e^{-\mu \rho \delta}\right) e^{-\mu \rho r}
$$

With a $\delta$, its approximate formula is expressed as:

$$
E=\Phi_{0} \mu e^{-\mu \rho r}
$$

The result of the deposition of mono-energetic photon energy is shown as above. Energy deposition requires the energy spectrum to be grouped according to the photon energy for X-ray with a certain energy spectrum (e.g., blackbody spectrum). Thus, the energy deposited in the unit mass material at $r$ can be expressed as:

$$
E=\sum_{i=1}^{N} \Phi_{0 i} \mu_{i} e^{-\mu_{i} \rho r}
$$

where $i$ denotes the photon cluster number, and $N$ is the number of clusters.

\section{Equivalent Analysis of Mono-Energy and Blackbody Spectrum X-ray}

The mono-energetic $X$-ray refers to an X-ray spectrum containing only one energy component. Given the characteristics of blackbody radiation, the simulation of the X-ray radiating effect of blackbody spectrum with several characteristic energies is of practical significance. $3 \mathrm{keV}$ and $5 \mathrm{keV}$ can more easily generate mono-energetic $X$-rays in experiments, while producing K-layer radiation of argon and titanium. Furthermore, the peak energy of $3 \mathrm{keV}$ approaches to $2.8 \mathrm{keV}$ blackbody spectrum, while $5 \mathrm{keV}$ is close to the mean value energy of $1.4 \mathrm{keV}$ blackbody spectrum $(4.9 \mathrm{keV})$. Thus, it is more practical to consider that $3 \mathrm{keV}$ and $5 \mathrm{keV}$ mono-energetic $X$-rays are equated with the $X$-ray impulse of $1 \mathrm{keV}$ and $1.4 \mathrm{keV}$ blackbody spectra.

Accordingly, mono-energetic X-rays of $3 \mathrm{keV}$ and $5 \mathrm{keV}$, and the blackbody X-rays of $1 \mathrm{keV}$ and $1.4 \mathrm{keV}$ were introduced in this study. There were two calculated mono-energetic and blackbody X-rays, respectively. The range of injection was $50 \sim 500 \mathrm{~J} / \mathrm{cm}^{2}$, the X-ray was a rectangular pulse of $0.1 \mathrm{~s}$ in width.

Figure 3 presents the impulse results of $3 \mathrm{keV}$ mono-energetic and $1 \mathrm{keV}$ blackbody $\mathrm{X}$-ray in different energy fluxes. As suggested from the results, the blow-off impulse rises monotonously with the energy flux in a certain range. Overall, the blow-off impulse of $3 \mathrm{keV}$ mono-energetic is larger than $1 \mathrm{keV}$ black body $\mathrm{X}$-ray, whereas that of $1 \mathrm{keV}$ black 
body is higher than that of $3 \mathrm{keV}$ mono-energetic in the case of high injection. Such result suggests a feasible way to simulate $1 \mathrm{keV}$ blackbody $\mathrm{X}$-ray irradiated blow-off impulse by $3 \mathrm{keV}$ mono-energetic $\mathrm{X}$-ray, and the numerical results of two types of $\mathrm{X}$-ray irradiated blow-off impulse were analyzed, and some numerical correlations were identified. On that basis, a simple numerical relationship of blow-off is given. The equivalent formula is written as:

$$
I_{k T=1}=\frac{6}{5} I_{h v=3}-60 \mathrm{~Pa} \cdot \mathrm{s}
$$

where $I$ denotes the blow-off impulse, $k T$ is the black body temperature and $h v$ is monoenergetic. This formula expresses the numerical relationship of blow-off impulse in the identical energy flux, which is termed as the equal energy flux relation. Figure 4 also draws the comparison between the fitting and numerical results.

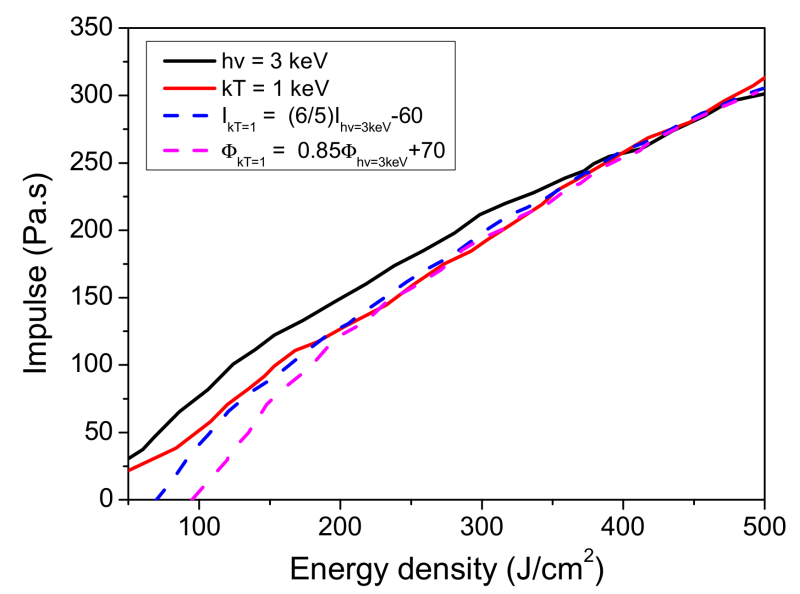

Figure 4. Comparison between the Mono-energetic $3 \mathrm{keV}$ and $1 \mathrm{keV}$ Black-Body Spectra.

It is inconvenient to apply the spectrum directly to equivalence and formulate the equivalent principle, though the equal energy flux relation is significantly simple. Thus, the relationships of two blow-off impulse dates were delved, and the relationship of energy flux in the identical blow-off impulse was given, which is termed as the equal blow-off impulse relation. The equivalent formula is written as:

$$
\Phi_{k T=1}=0.85 \Phi_{h v=3}+70 \mathrm{~J} / \mathrm{cm}^{2}
$$

where $\Phi$ denotes the energy flux. This formula gives the energy flux of $1 \mathrm{keV}$ blackbody spectrum X-ray in the identical blow-off impulse, and the equivalent relationship can be determined. Figure 3 also illustrates the equal blow-off impulse relation, and the two curves show consistency above $200 \mathrm{~J} / \mathrm{cm}^{2}$, and a big difference was identified in low energy flux. From equal energy flux relation and equal blow-off impulse relation, it is suggested that the relations were self-consistent and complete above $200 \mathrm{~J} / \mathrm{cm}^{2}$.

Overall, the range of anti-radiation reinforcement parameter was $120 \sim 300 \mathrm{~J} / \mathrm{cm}^{2}$, so this study placed more emphasis on the mentioned results in this range. To solve the error problem of low energy flux, a new equal-impulse relation is fitted for the anti-radiation reinforcement energy injection quantity index. The equivalent formula is presented as:

$$
\Phi_{k T=1}=\Phi_{h v=3}+35 \mathrm{~J} / \mathrm{cm}^{2}
$$

The X-ray blow-off impulse of $1 \mathrm{keV}$ blackbody spectrum given by the equation is very close to the numerical calculation result, whereas the results were very different in the high energy flux above $300 \mathrm{~J} / \mathrm{cm}^{2}$. The equal impulse relation should be adopted according to the actual data, as shown in Figure 5. 


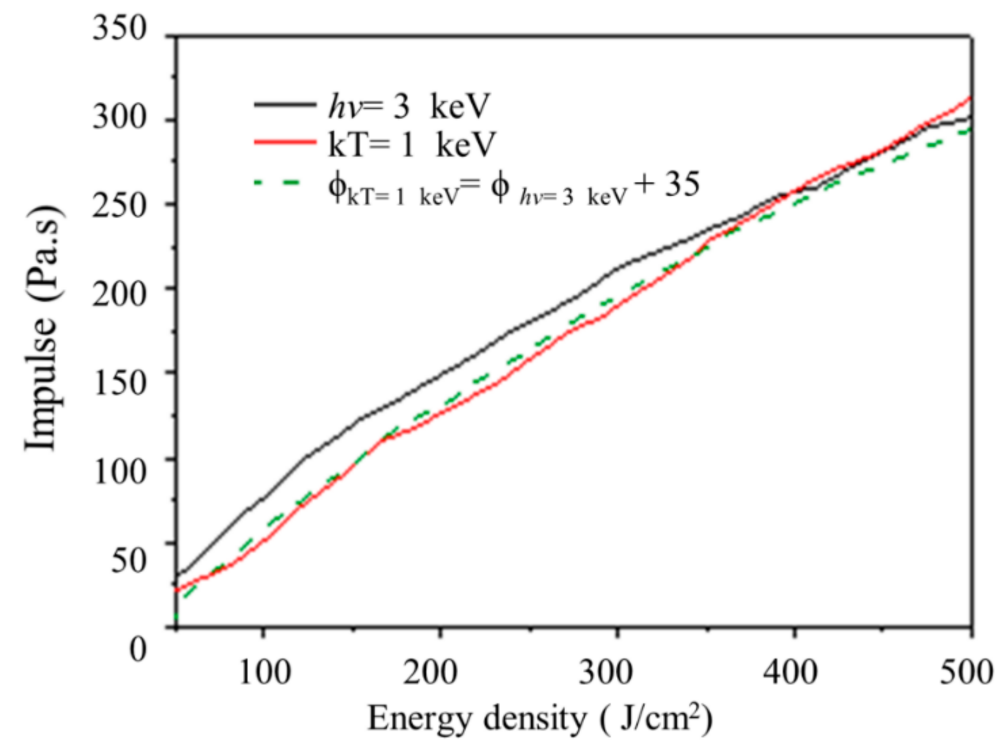

Figure 5. Equal impulse relation of $1 \mathrm{keV}$ blackbody spectrum under anti-radiation reinforcement parameter.

The blow-off impulse result between $5 \mathrm{keV}$ mono-energetic and $1.4 \mathrm{keV}$ blackbody X-rays is given in Figure 6, in which the blow-off impulse monotonously increased with energy flux in a certain range of flux. This trend is different from that in Figure 2, due to the difference in energy deposition profile caused by different $X$-ray energy spectrum distributions. The impulse of $5 \mathrm{keV}$ mono-energetic $X$-ray was greater than that of $1.4 \mathrm{keV}$ blackbody X-ray, and such difference increased with the increase in energy injection, the maximum difference was nearly $150 \mathrm{~Pa} \cdot \mathrm{s}$.

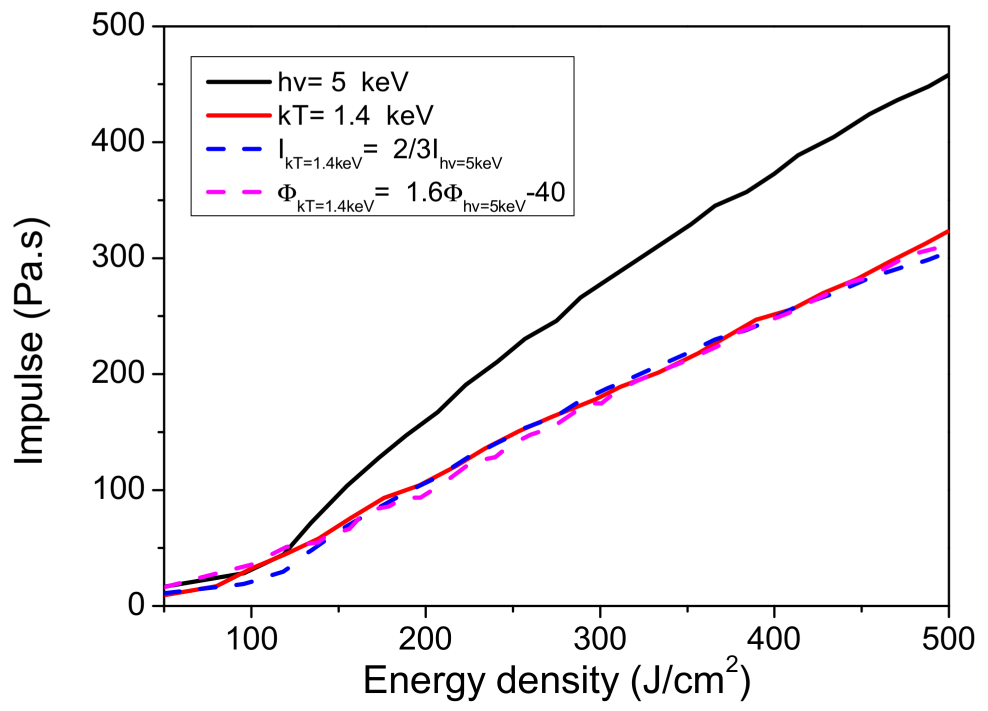

Figure 6. Comparison between the $5 \mathrm{keV}$ mono-energetic and $1.4 \mathrm{keV}$ blackbody spectra.

The results of blow-off impulse of two types of X-ray were analyzed, and the equivalent blow-off relation was given. The equivalent formula is written as:

$$
I_{k T=1.4}=\frac{2}{3} I_{h v=5} \mathrm{~Pa} \cdot \mathrm{s}
$$

Figure 6 presents the blow-off impulse results of the numerical and $1.4 \mathrm{keV}$ blackbody spectrum from the equal energy flux relation, complying with the range of energy flux. The 
energy flux equivalent relationship of equal impulse relation for $5 \mathrm{keV}$ mono-energetic and $1.4 \mathrm{keV}$ blackbody spectrum was given. The formula is written as:

$$
\Phi_{k T=1.4}=1.6 \Phi_{h v=5}-40 \mathrm{~J} / \mathrm{cm}^{2}
$$

Figure 7 also gives the numerical results of $5 \mathrm{keV}$ mono-energetic and $1.4 \mathrm{keV}$ blackbody spectrum, as well as the fitting curve of equal blow-off impulse and equal energy flux relation. They were consistent within $500 \mathrm{~J} / \mathrm{cm}^{2}$, revealing that the two relationships were self-consistent and complete.

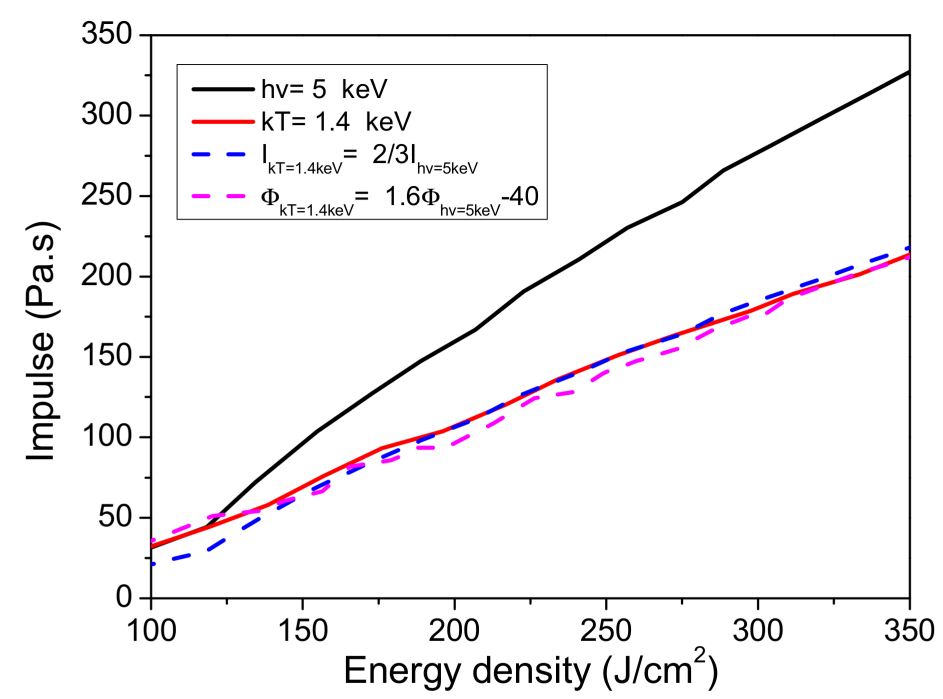

Figure 7. Comparison between $5 \mathrm{keV}$ mono-energetic and $1.4 \mathrm{keV}$ blackbody $\mathrm{X}$-rays under antiradiation reinforcement parameter.

Figure 7 also shows the relationship between the numerical calculation results and the X-ray blow-off impulse of $1 \mathrm{keV}$ blackbody spectrum calculated by two formulas within the radiation reinforcement energy injection range, and the results are satisfactory. Figures 8 and 9, respectively, show equal-energy fluence curves on the impulse plane and equal-impulse curves on the energy injection plane obtained by the equal-energy fluence relationship and equal-impulse relationship. Even though the curves are basically a straight line, the equal energy flux curve and equal impulse curve should be a non-linear curve, and mathematical expressions were presented. In fact, the equal-energy fluence curve and equal-impulse curve are a nonlinear curve, with their mathematical expressions determined by numerical calculation or experimental results or both. The point above the curve shows that the energy flux of the blackbody spectrum is greater than the mono-energetic $\mathrm{X}$-ray under the corresponding impulse, whereas the point on the curve equals to the blackbody spectrum and the mono-energetic. The blow-off impulse of the blackbody spectrum is greater than that of the mono-energetic in the upper region of the equal impulse curve, exactly equal to them on the curve, but it is opposite underneath the curve. It lays the basis for blow-off impulse equivalence of the equal blow-off impulse relation and the equal energy flux relation, and they are mutually complemented and confirmed. It reflects the law of equivalence from different angles and aspects in the study on blow-off impulse equivalence, and the characteristics of self-consistency and completeness are presented. The equal blow-off impulse relation and the equal energy flux relation can be exploited as the method of equivalent blow-off impulse, which will be critical to the equivalent simulation of X-ray irradiation blow-off impulse. 


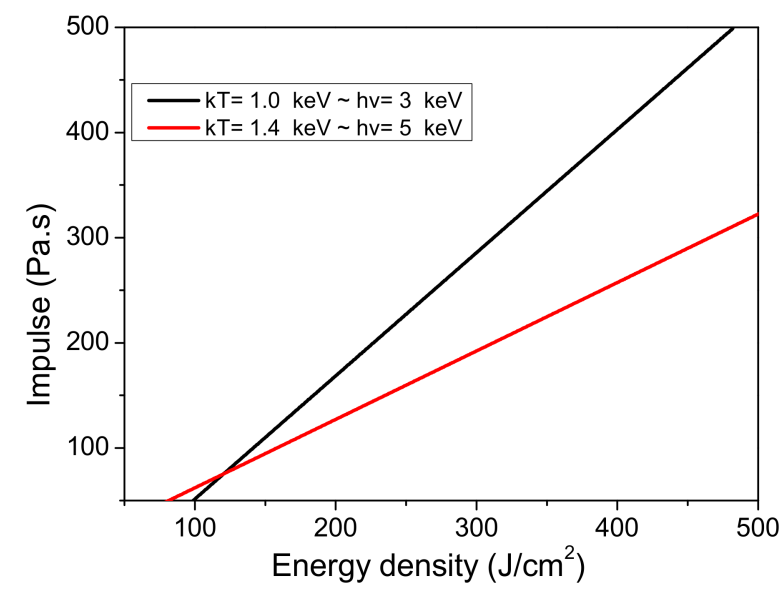

Figure 8. Equal energy flux curve on impulse plane.

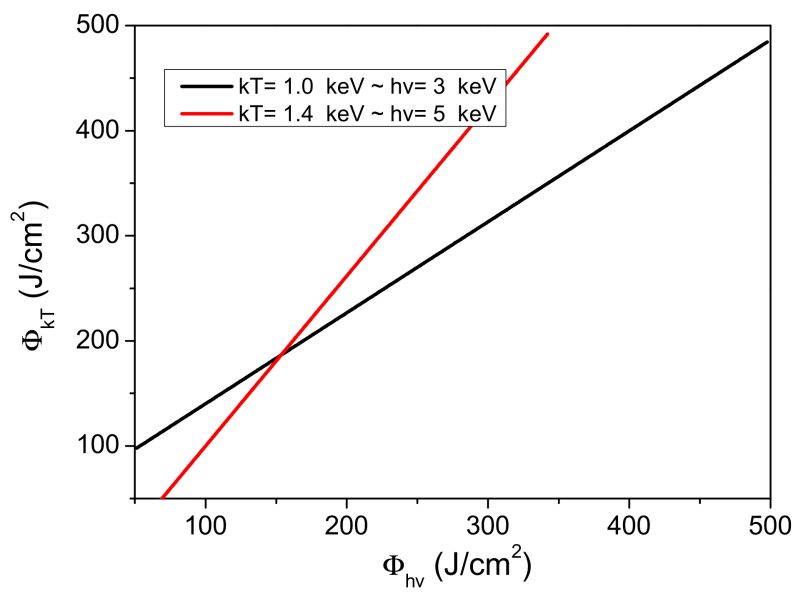

Figure 9. Equal impulse curve on energy flux plane.

In this study, the results of blow-off impulse of aluminium alloy material, i.e., irradiated X-ray of $1 \mathrm{keV}$ or $1.4 \mathrm{keV}$ blackbody spectra and $3 \mathrm{keV}$ or $5 \mathrm{keV}$ mono-energetic, were simulated and analyzed. The equal energy flux relationship and equal impulse relationship of X-ray impulse were characterized with mono-energetic X-ray equivalent blackbody spectrum for aluminium materials, and the concept of equal energy flux curve and equal impulse curve was developed, and the equivalent method to simulate $X$-ray radiation blow-off impulse was proposed. The mentioned information supported the laboratory simulation of blow-off impulse of X-ray.

\section{Effect of X-ray Angle of Incidence of Blow-Off Impulse}

When a parallel X-ray irradiates the structure or material surface, there will be different angles at different directions for the normal structure surface and radiated direction of $\mathrm{X}$-ray. It directly affects the distribution of X-ray energy fluxes on the surface, and the distribution law of blow-off impulse, and the load of different distribution directly affects the structural response law. Accordingly, it is of great significance to study the effect of $\mathrm{X}$-ray angle of incidence of blow-off impulse.

No definite conclusion has been drawn about the distribution of X-ray blow-off impulse with the angle. When Cost [1,4] calculated the impulse of cylindrical shell by X-ray, the X-ray on the surface of cylindrical shell is in fact cosine distribution with the angle of incidence. Thus, it was intuitively assumed that the blow-off impulse is cosine distribution, whereas there is no corresponding proof and applicable conditions. Subsequently, researchers in this field have been exploiting this hypothesis to the load design of structural response laboratory simulation $[2,3,5,6]$. However, the relation of blow-off impulse with 
the angle of incidence has been explored. A clear conclusion from analytical deduction and derivation is difficult to draw since formula is overly sophisticated about X-ray energy deposition of blackbody spectrum. Cost conducted an analytical deduction of the energy deposition on the surface of cylindrical shells irradiated by X-ray in different directions [4], and the conclusion was also unsatisfactory. He also calculated and analyzed the blow-off impulse of cylindrical shell by X-ray, whereas the numerical results cannot account for the problem since the relation is not the same between the different direction of two-dimensional mesh and the incidence direction of X-ray. Thus, there has been no effective progress in the study on the relation between the pulsed X-ray blow-off impulse and the angle of incidence over the past few years.

Since the problem originates from the pulsed X-ray effect of two-dimensional cylindrical shells, the method was also illustrated in the two-dimensional framework, thereby to some extent limiting the solution. In this study, the oblique incidence of $\mathrm{X}$-ray was discussed in a two-dimensional frame, and the relation between the pulsed X-ray blow-off impulse and the angle of incidence was obtained by numerical calculation. Figure 10 presents the oblique incidence model of X-ray; the dotted line is in perpendicular incident direction, while the solid line is in oblique incident direction. From the geometry in the figure, the energy deposition thickness of X-ray in the identical size and location of the grid can be concluded. The formula is written as:

$$
\Delta R=\frac{\Delta r}{\cos \theta}
$$

where $\Delta R$ denotes the energy deposition thickness of oblique incident $\mathrm{X}$-ray in the grid, $\Delta r$ is the energy deposition thickness of vertical incident $X$-ray and $\theta$ represents the angle between the incident $X$-ray direction and the normal direction of the target surface.

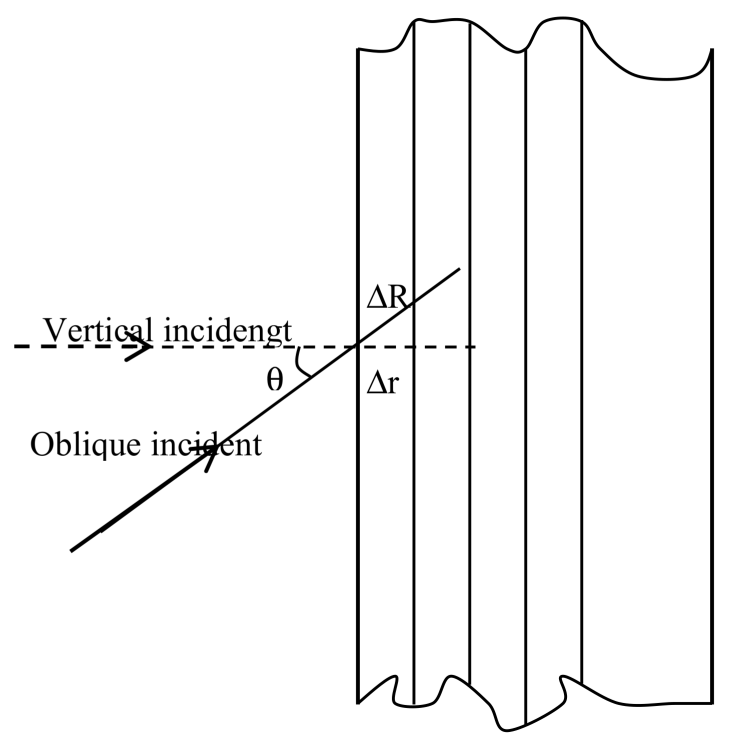

Figure 10. Geometric relation between oblique incidence of X-ray and computational mesh.

For mono-energetic $\mathrm{X}$-ray irradiation, the energy deposition at $r$ can be deduced as:

$$
E=\Phi_{0} \mu e^{-\frac{-\mu \rho r}{\cos \theta}}
$$

Since material melting is an essential condition to blow-off impulse. $E_{v}$ is the melting energy of material, so the thickness of melting layer can be computed from the above formula. The formula is written as:

$$
r_{v}=\frac{\cos \theta}{\mu \rho} \ln \frac{\Phi_{0} \mu}{E_{v}}
$$


The melt thickness of the material is basically cosine distribution with angle of incidence for mono-energetic $\mathrm{X}$-ray. Blow-off impulse is expressed as:

$$
I=\int_{0}^{r_{v}} u \rho d r
$$

where $u$ denotes the particle velocity. The numerical calculation indicates that the particle velocity is basically the same when the energy deposition is similar in the material. Accordingly, the blow-off impulse was determined by the melting thickness of the material. Thus, the blow-off impulse distribution is basically cosine for mono-energetic $\mathrm{X}$-ray. The blackbody spectrum X-ray is composed of a series of mono-energetic X-rays, so a simple and intuitive formula similar with Formulas (47) and (48) is difficult to obtain. It is simulated the blow-off impulse blackbody spectrum X-ray at different angles of incidence in one-dimensional model by the self-developed software.

The typical material parameters of aluminium alloy are selected as input condition, and the blackbody spectrum were the selected soft one with blackbody temperature of $1 \mathrm{keV}$ or $1.4 \mathrm{keV}$ and hard spectrum with $3 \mathrm{keV}$ and $5 \mathrm{keV}$, and X-ray time spectrum was rectangular pulse of $0.1 \mathrm{keV}$ in width. The variation of blow-off impulse with the angle of incidence of X-ray was, respectively, calculated according to four X-ray spectra, and the range of X-ray energy flux was $100-500 \mathrm{~J} / \mathrm{cm}^{2}$.

Figure 11 shows the calculated results of the blow-off impulse of soft $X$-ray at different angles of incidence. A simple fitting formula of soft X-ray blow-off impulse with the angle of incidence is obtained. The cosine distribution formula is as follows.

$$
I_{\theta}=I_{0} \cos \theta
$$

where $I_{\theta}$ is the blow-off impulse of X-ray with an angle of incidence $\theta$, and $I_{0}$ is the impulse of $X$-ray vertical angle of incidence. It is therefore suggested that the blow-off impulse is basically cosine distribution for soft X-ray, thereby verifying the cosine distribution hypothesis from calculation. The point represents the calculation result in the figure, and the solid line represents the result of fitting.
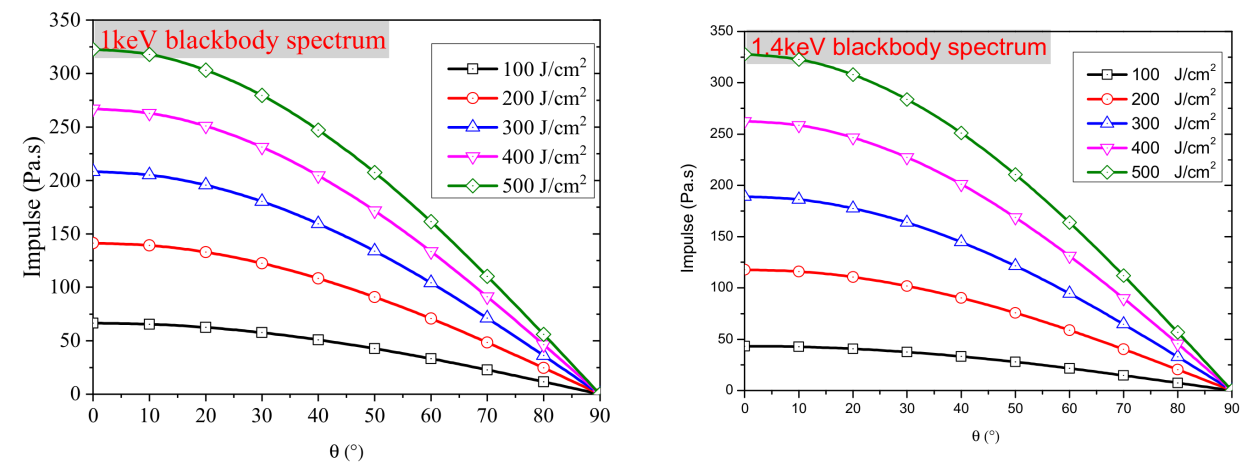

Figure 11. Relation between blow-off impulse and angle of incidence of soft X-ray.

Figure 12 shows the calculated results of blow-off impulse of hard X-ray at different angles of incidence. The results deviate from cosine distribution for hard X-ray, whereas it still satisfies cosine distribution in a certain angle range. A fitting formula of the hard X-ray blow-off impulse with the angle of incidence is obtained. The cosine distribution formula is presented as:

$$
I_{\theta}=I_{0} \cos (1.15 \theta)
$$



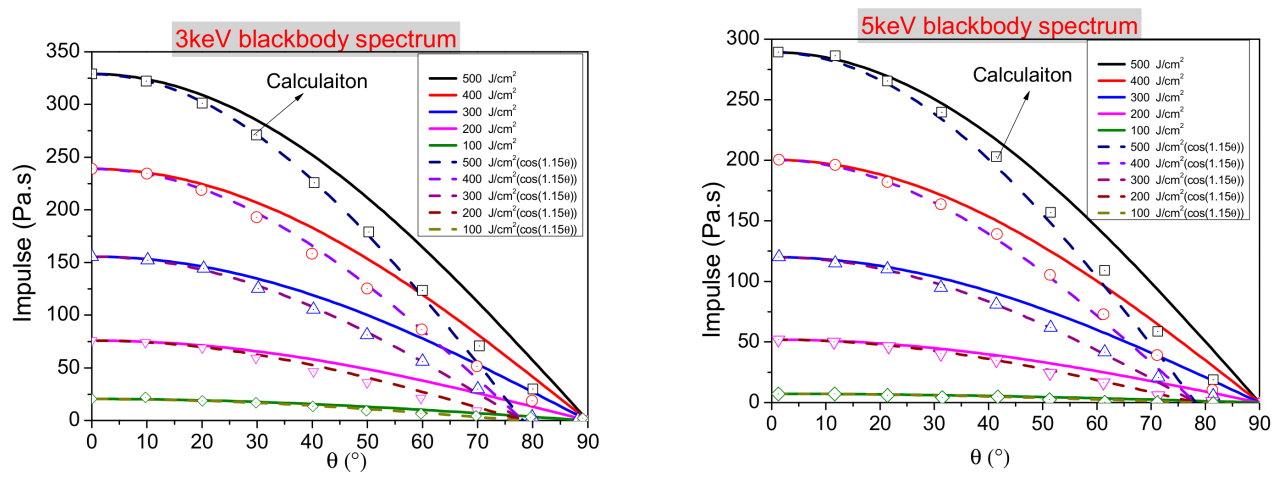

Figure 12. Relation between blow-off impulse and angle of incidence of hard X-ray irradiation.

It is suggested that the fitting formula is well consistent with the numerical results in the range of $70^{\circ}$.

\section{Responses of Cylindrical Shell Loaded LIHE to Simulate X-ray Effects}

Intense soft (cold) X-ray blow-off impulses induced by atmospheric nuclear explosions will lead to structural responses and consequently may even damage or destroy flying re-entry structures. Therefore, dynamic structural responses of typical structures are significantly necessary to be investigated for weapon design and validation. Without actual nuclear explosion or X-ray radiation environments, some test simulation techniques are used to generate equivalent impulsive loads, this study focuses on Light Initiated High Explosive (LIHE) method and numerically investigates the fidelity of test simulation.

A typical impulse induced by X-ray blow-off can be given as Figure 13. On the surface of the axisymmetric structure, the impulse is cosine-distributed around the circumference, and uniformly distributed along the outer generating lines. The basic concept of LIHE technique and experimental photograph is illustrated as Figure 14. The load, i.e., the cosine-distributed specific impulse, is meshed into finite elements using generating lines (Spray process causes). In addition, the impulse in each element is simulated by the LIHE.
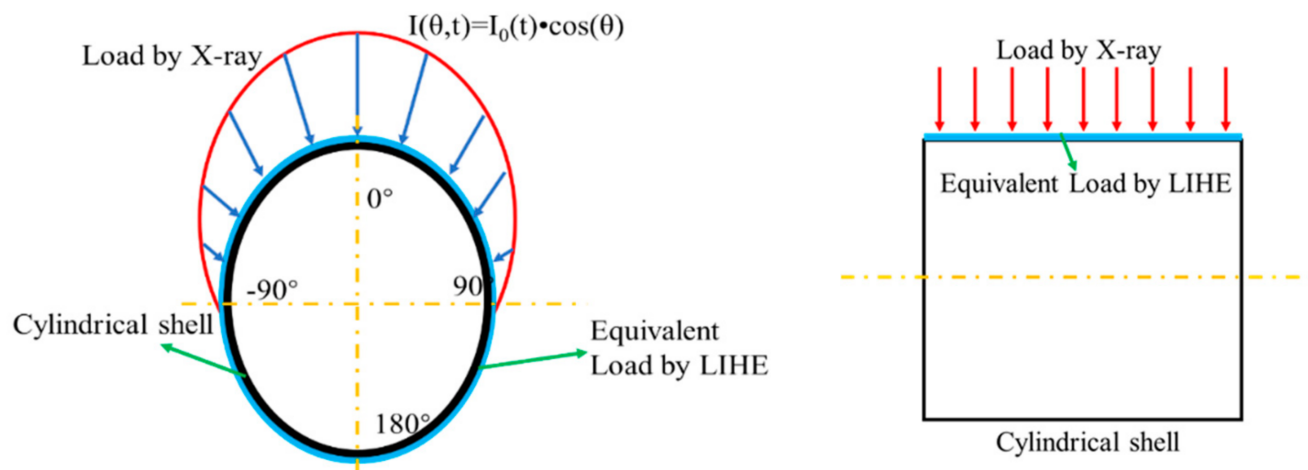

Figure 13. X-ray blow-off impulse model on the surface of a cylindrical shell.

The aluminum alloy cylinder shell was selected as the experimental object. The inner diameter of the cylinder shell was $150 \mathrm{~mm}$, the wall thickness was $3 \mathrm{~mm}$ and the length was $180 \mathrm{~mm}$. During the experiment, one end of the cylinder shell was lifted and presented an approximate free state, as shown in Figure 15. The photosensitive explosive is distributed within the range of $\pm 90^{\circ}$. The pulsed flashlight source is used to initiate the metal wire to generate strong light and synchronously initiate the photosensitive explosive. The peak value of specific impulse designed in the experiment is $150 \mathrm{~Pa} \cdot \mathrm{s}$, which is evenly distributed along the bus direction of the cylinder shell, and the circumferential direction is cosine distribution to complete the lateral load loading of the cylinder shell. 

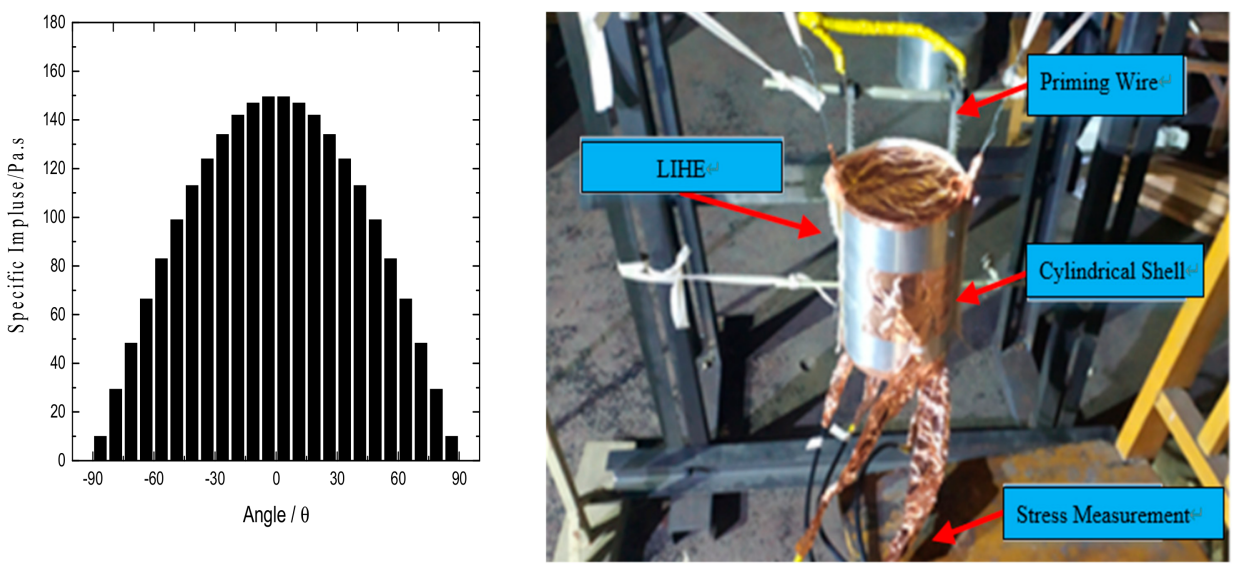

Figure 14. Illustration of the superposition method and experimental photograph.

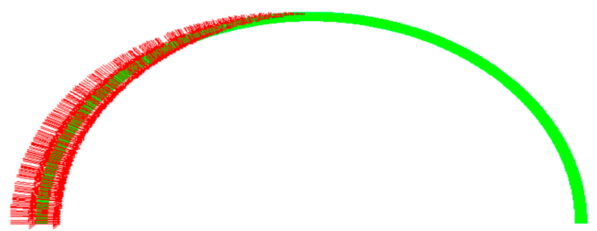

Figure 15. Finite element model of the cylindrical shell.

The model was built in Dyna/CAE. A $\Phi 150 \mathrm{~mm} \times 180 \mathrm{~mm}$ and $3 \mathrm{~mm}$-thick cylindrical shell is considered. The material, assumed as Alloy, is characterized by linear elastic constitutive relationship. The density, Young's modulus and Poisson's ratio are $2.7 \times 10^{3} \mathrm{~kg} / \mathrm{m}^{3}$, $72 \mathrm{GPa}$ and 0.35 , respectively. The $1 / 2$ model is meshed into 360 (circumferential direction) $\times 21$ (axial direction) $\times 4$ (thickness direction) $=30,240$ C3D8R elements, as shown in Figure 15.

Figure 16 gives the statistics of the peak stress errors and shows the shapes of stress histories hardly vary in the mass, but the peaks change a little, the maximum error of stress peak keeps less than $8 \%$. So, the influence of non-simultaneous initiation on simulation fidelity is rather significant. Thus, in practical tests, the detonating LIHE should be insured in a cosine thickness as possible as we can.

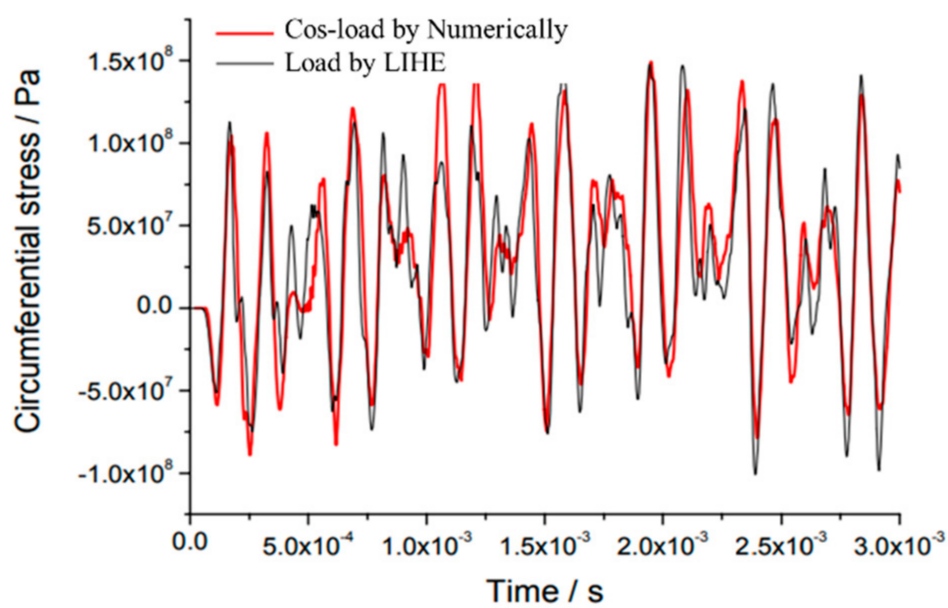

Figure 16. The statistics of the peak stress errors between experimental and numerically.

\section{Conclusions}

This study discussed blow-off impulse equivalent of mono-energetic and blackbody spectra X-ray, point source and parallel X-ray, and drew the following conclusions: 
(1) The blow-off impulse of blackbody spectrum can be well simulated by the peak energy and the mean value energy of mono-energetic X-ray. Equivalent blackbody spectrum X-ray blow-off impulse is theoretically feasible based on the characteristic energy of mono-energetic $\mathrm{X}$-ray.

(2) It gives the equal energy flux relation of blow-off impulse between mono-energetic and blackbody spectra for aluminium alloy materials. Two formulas are well consistent with each other in the range of energy flux. It can estimate blow-off impulse of blackbody X-ray under equal energy flux by the result of mono-energetic, and the indirect experimental data were provided for reinforcement technology and evaluation of reinforcement efficiency.

(3) It gives the equivalent energy flux relationship under equal impulse of mono-energetic and blackbody spectra of aluminium materials. Two formulas also comply with each other in the range of energy flux. The mono-energetic $\mathrm{X}$-ray impulse experiment is designed to simulate the blackbody spectrum $X$-ray impulse experiment under specific energy flux by equal impulse relation. It is equivalent to provide direct experimental data for reinforcement technology and assessment of reinforcement efficiency.

(4) Equal energy flux curve and equal impulse curve exhibited their equivalent relationship from their formula, which can be critical to the equivalent simulation of X-ray blow-off impulse.

(5) A relatively complete method of equivalent was provided, with the mono-energetic simulating blow-off impulse of intense pulse blackbody spectrum X-ray by equal energy flux and equal impulse relation. Absolute mono-energetic parameters of simulation were used, whereas they were difficult to produce in experimental equipment. Thus, it can be more feasible that two relationships are established for the blow-off impulse results of numerical simulation based on mono-energetic X-ray measured spectrum.

(6) The simulated parallel X-ray by point source can be basically satisfied with the nonuniformity experimental requirements of energy flux. Furthermore, the equivalent experiment of X-ray thermo-dynamic effect of plate target can be carried out.

(7) The equivalent of structural response between point source and parallel $\mathrm{X}$-rays is uncertain and it has a big difference in energy flux distribution.

(8) The theoretical analysis and numerical simulation of blow-off impulse of oblique incidence of pulsed blackbody spectrum $X$-ray by the one-dimensional model were conducted in this study. It was preliminarily verified that the assumption of cosine distribution is feasible for soft X-ray irradiation, and some deviation for hard X-ray irradiation was demonstrated. The mentioned findings laid a technical basis for the load design of structural response simulation experiment of pulsed X-ray. The discussion was limited to hard aluminium materials and $1 \mathrm{keV} \sim 5 \mathrm{keV}$ blackbody $\mathrm{X}$-ray, and it should be studied in depth to draw more extensive conclusions.

(9) LIHE simulation techniques are used to generate equivalent impulsive loads with actual nuclear explosion or X-ray radiation environments. The peaks change a little between experimental and numerically, the maximum error of stress peak keeps less than $8 \%$.

Author Contributions: Conceptualization, D.W.; methodology, J.Z.; validation, D.W. and W.C.; formal analysis, D.W. and Y.G.; investigation, D.W., J.Z. and Y.G.; data curation, D.W. and Y.G.; writing—original draft preparation, D.W.; writing—review and editing, D.W., Y.G. and S.W.; supervision, S.W. All authors have read and agreed to the published version of the manuscript.

Funding: This work was supported by the key project of Intergovernmental International Scientific and Technological Innovation Cooperation in China under Grant No. 2016YFE0128900, and the National Natural Science Foundation of China under Grant No. 11775166.

Institutional Review Board Statement: Not applicable.

Informed Consent Statement: Not applicable. 
Data Availability Statement: All data included in this study are available upon request by contact with the corresponding author.

Conflicts of Interest: The authors declare no conflict of interest.

\section{References}

1. Office of the Deputy Assistant Secretary of Defense for Nuclear Matters (ODASD(NM)). Nuclear Matters Handbook. 2020.

2. Glasstone, S.A. The Effects of Nuclear Weapons (Revised Edition); United States Atomic Energy Commission: Washington, DC, USA, 1977; pp. 12-27, 671-677.

3. Johansson, A. Principles and techniques of radiation hardening. Nucl. Instrum. Methods 1977, 140, 408. [CrossRef]

4. Osigwe, E.O.; Gad-Briggs, A.; Nikolaidis, T.; Jafari, S.; Sethi, B.; Pilidis, P. Thermodynamic Performance and Creep Life Assessment Comparing Hydrogen- and Jet-Fueled Turbofan Aero Engine. Appl. Sci. 2021, 11, 3873. [CrossRef]

5. Lawrence, R.J. The Equivalence of Simple Models for Radiation-Induced Impulse; Elsevier: Amsterdam, The Netherlands, 1992; pp. 785-788.

6. Cost, T.L. Dynamic Response of Missile Structures to Impulsive Loads Caused by Nuclear Effects Blow Off. 1976.

7. Qiu, A.C. The development of technology for pulsed X-ray simulators. Eng. Sci. 2000, 2, $24-28$.

8. Chen, Z.L.; Peng, S.M.; Meng, D.; He, Y.H.; Wang, H.Y. Monte Carlo calculation of energy deposition in ionization chambers for tritium measurement. Nucl. Instrum. Methods Phys. Res. A 2014, 762, 7-10. [CrossRef]

9. Wu, D.; Xu, X.; Zhang, L.; Wang, S. A hybrid Monte Carlo model for the energy response functions of X-ray photon counting detector. Nucl. Instrum. Methods Phys. Res. A 2016, 83, 397-406. [CrossRef]

10. Benham, R.A. Reentry Vehicle Cold X-ray Induced Impulse Technology Development: Materials Blow-Off Characteristics, Modelling, and Experimental Facilities; Sandia National Laboratories: Albuquerque, NM, USA, 1997; SASN1997-2023.

11. Rivera, W.G.; Benham, R.A. Explosive Technique for Impulse Loading of Space Structures; Sandia National Laboratories: Albuquerque, NM, USA, 1972; SASN 1999-3175.

12. Briggs, E.A.; Veigele, W.J. X-ray Cross Section Compilation from $0.1 \mathrm{keV}$ to $1 \mathrm{MeV}$; DNA 2433; Kaman Sciences Corporation: Colrado Springs, CO, USA, 1972; Volume 1.

13. Liu, Y.; Hu, X. Kinetics and Thermodynamics of Efficient Phosphorus Removal by a Composite Fiber. Appl. Sci. 2019, 9, 2220. [CrossRef]

14. Karakas, A.; Tunc, M.; Camdali, U. Thermal analysis of thin multi-layer metal films during femtosecond laser heating. Heat Mass Transf. 2010, 46, 1287-1293. [CrossRef]

15. Hettche, L.R.; Tucker, T.R.; Schrempf, J.T. Mechanical response and thermal coupling of metallic targets to high-intensity 1.06- $\mu \mathrm{m}$ laser radiation. J. Appl. Phys. 1976, 47, 1415-1421. [CrossRef]

16. Doron, K.; Eli, W. Hard X-ray emission from accretion shocks around galaxy clusters. J. Cosmol. Astropart. Phys. 2010, 2, $219-231$. [CrossRef]

17. Zhu, J.; Yu, H.; Chen, N.; Jiang, X.; Zhang, Z.; Dai, W.; Wang, Y.; Wang, X.; Wang, Y.; Wang, J.; et al. Hydrodynamic response of converter target impacted by high current relativistic electron beam. Nucl. Instrum. Methods Phys. Res. Sect. B 2011, 269, 2139-2144. [CrossRef]

18. Bifulco, A.; Parida, D.; Salmeia, K.; Nazir, R.; Lehner, S.; Stämpfli, R.; Markus, H.; Malucelli, G.; Branda, F.; Gaan, S. A review of multi-physical fields induced phenomena and effects in spark plasma sintering: Fundamentals and applications. Mater. Des. 2020, 191, 108-120. [CrossRef]

19. Sakamiti, G.P.; de Siqueira, R.H.M.; de Carvalho, S.M.; Meireles, J.B.; de Lima, M.S.F. Weldability of a zirconium alloy comparing resistance and pulsed laser methods. Nucl. Mater. Energy 2019, 20, 100693. [CrossRef]

20. Li, M.; Sommerer, M.; Werner, E.; Lampenscherf, S.; Steinkopff, T.; Wolfrum, P.; You, J.-H. Experimental and computational study of damage behavior of tungsten under high energy electron beam irradiation. Eng. Fract. Mech. 2015, 135, 64-80. [CrossRef]

21. Report of the Defense Science Board Task Force on Nuclear Weapon Effects Test, Evaluation, and Simulation; Defense Science Board (DSB): Washington, DC, USA, 2005.

22. Yang, H.; Qiu, A.; Zhang, J.; Huang, J.; Sun, J. Simulation calculation for the energy deposition profile and the transmission fraction of intense pulsed electron beam at various incident angles. High Power Laser Part. Beams 2002, 14, 778-782.

23. Hu, Y.; Yang, H.L.; Sun, J.F.; Sun, J.; Zhang, P.F. Faraday cup array designed for measurement of intense pulsed electron beam incident angles. Prog. Rep. China Nucl. Sci. Technol. 2015, 4, 18-23.

24. Qiao, D.J. Thermodynamic Effect and Reinforcing Technology under Pulse X-ray Radiation; National Defense Industry Press: Beijing, China, 2012; pp. 118-196.

25. Qiao, D.J.; Zhou, N. Materials Dynamics under Pulse Beam Radiation; National Defense Industry Press: Beijing, China, 2002; pp. 1-56.

26. Venkata, K.A.; Truman, C.E.; Smith, D.J.; Bhaduri, A.K. Characterizing electron beam welded dissimilar metal joints to study residual stress relaxation from specimen extraction. Int. J. Press. Vessel. Pip. 2016, 139, 237-249. [CrossRef]

27. Leggieri, A.; Passi, D.; Di Paolo, F.; Spataro, B.; Dyunin, E. Design of a sub-millimetric electron gun with analysis of thermomechanical effects on beam dynamics. Vacuum 2015, 122, 103-116. [CrossRef]

28. Sun, J.; Hu, Y.; Sun, J.; Yang, H.; Zhang, P.; Jin, L. An average method for intense pulsed electron beam incident angles. High Power Laser Part. Beams 2015, 27, 140011-140014. [CrossRef] 
29. Lupu-Sax, A. S Quantum Scattering Theory and Applications. Ph.D. Thesis, Harvard University, Cambridge, MA, USA, 1998; pp. 19-64.

30. Yang, H.; Hai-Liang, Y.; Jian-Feng, S.; Jiang, S.; Peng-Fei, Z. A method of measuring the incidence angle of intense electron beam. Acta Phys. Sin. 2015, 64, 245203. [CrossRef]

31. Milov, I.; Lipp, V.; Ilnitsky, D.; Medvedev, N.; Migdal, K.; Zhakhovsky, V.; Khokhlov, V.; Petrov, Y.; Inogamov, N.; Semin, S.; et al. Similarity in ruthenium damage induced by photons with different energies: From visible light to hard X-rays. Appl. Surf. Sci. 2020, 501, 143973. [CrossRef]

32. Hu, L.; Lei, Y.; Zhu, J. Simulation on distributed target material impacted by high intensity current multi-pulse electron beam. High Power Laser Part. Beams 2013, 25, 2024-2130. [CrossRef]

33. Chavez, A.M.; Todd, T. Synthesis microstructure and explosive properties of spray-deposited silver acetylide-silver nitrate composite light initiated high explosives. In Proceedings of the 83rd Shock and Vibration Exchange, New Orleans, LA, USA, 4-8 November 2012. SAND2013-3088C.

34. Chavez, M.A. Implications of Explosively Accelerating Thin Flyer Plates in the Transient Regimes of Explosive Systems: A Thesis. Ph.D. Thesis, New Mexico Institute of Mining and Technology, Socorro, NM, USA, 2012. 